\title{
Multi-Phase Equilibrium in Aqueous Solutions of the Triple-Helical Polysaccharide, Schizophyllan
}

\author{
Takashi Itou and Akio Teramoto \\ Department of Macromolecular Science, Osaka University, \\ Toyonaka, Osaka 560, Japan
}

(Received June 11, 1984)

\begin{abstract}
Ternary phase diagrams were determined at $25^{\circ} \mathrm{C}$ for aqueous solutions of a rodlike polysaccharide schizophyllan, using three fractionated samples of different axial ratios $x$ : sample T-3, $x=223$; sample UR-28, 36.8; sample U-110, 18.3. Two ternary systems UR-28+ $\mathrm{T}-3+$ water and $\mathrm{U}-110+\mathrm{T}-3$ + water were investigated. The phase diagram of either system was occupied by a wide biphasic region, where a dilute isotropic phase and concentrated cholesteric phase coexisted at equilibrium. The phase diagram of the system U-110+T-3+water contained two additional regions, one where two cholesteric phases and one isotropic phase coexisted at equilibrium and the other where two cholesteric phases coexisted. A remarkable molecular weight fractionation took place on phase separation, and the larger $x$ component was almost excluded from the dilute phase (or phases). All these features of the phase diagrams were consistent with the theoretical prediction by Abe and Flory for athermal ternary solutions. Quantitative agreement between theory and experiment was far from satisfactory, although the disagreement was considerably diminished when the interaction parameter was incorporated into the Abe-Flory theory.
\end{abstract}

KEY WORDS Phase Equilibrium / Ternary System / Rodlike Polymer / Polysaccharide / Molecular Weight Fractionation / Triphase Separation / Cholesteric Liquid Crystal / Schizophyllan /

Recently, we have been interested in aqueous solutions of the polysaccharide, schizophyllan, ${ }^{1-4}$ which exists as a trimer forming a rigid straight helix in water. ${ }^{5,6}$ One purpose of these studies has been to investigate the phase behavior of solutions of rodlike polymers with schizophyllan as a model substance. Aqueous schizophyllan shows various interesting characteristics of rodlike polymers. Indeed, it forms a cholesteric mesophase above a certain critical concentration, and there is a concentration range where the isotropic and cholesteric phases coexist at equilibrium..$^{1-3}$ Detailed experimental data for this equilibrium compare favorably with existing theories of rodlike polymers ${ }^{7-9}$ in qualitative terms, although a quantitative agreement between theory and experiment has not yet been achieved. ${ }^{3}$

It was shown in a preliminary $\operatorname{paper}^{10}$ that an aqueous solution of two fractionated samples at appropriate composition separated into three phases at equilibrium: an isotropic phase and two cholesteric phases. This may be the first evidence for the theory of Abe and Flory $^{11}$ on ternary systems of two rodlike polymers in a single solvent. This theory as well as many others is formulated for monodisperse polymers, but most of the experimental systems consist of polydisperse polymers. As has been predicted theoretically ${ }^{7-9,11-15}$ and confirmed experimentally, ${ }^{3,16-22}$ the phase behavior of a given system depends on the molecular weight of the polymer solute, and hence on the molecular weight distribution for a polydisperse solute. Therefore, any experimental test of the theories cannot be conclusive unless due attention is paid on the samples' polydispersity. 
Following the previous work, ${ }^{10}$ we carried out phase separation experiments using two pairs of fractionated samples of schizophyllan differing in molecular weight by a factor of about 6 and 12, with particular attention directed to the samples' polydispersity and their effects on phase behavior. In this study, all the present and previous results are combined for a comparison with the theoretical prediction by Abe and Flory. The experimental details, major experimental findings, and conclusions from these findings are described in the following.

\section{EXPERIMENTAL}

\section{Polysaccharide Samples}

Sonicated schizophyllan samples supplied by Taito Co. were repeatedly fractionated by fractional precipitation with water as the solvent and ethanol or acetone as the precipitant. Fractions with appropriate molecular weights were collected and subject to gel filtration with water as the eluent; Sepharose CL-2B was used for the highest molecular weight fractions and Sepharose CL-6B for the rest. Appropriate middle fractions were combined to obtain three samples: T-3, UR-28, and U-110; sample U-110 was the polymer recovered from the cholesteric phase separated from a biphasic mixture of the combined lowest molecular weight fractions. ${ }^{23}$

Molecular weights of the samples were determined from sedimentation equilibrium measurements according to the previous procedure. ${ }^{3}$ The samples were also analyzed by gel permeation chromatography (GPC) by a Toyo Soda HLC-803D instrument equipped with refractive index (RI) and light scattering (LS) detectors. The operating conditions were as follows: TSK-Gel G6000PW and G5000PW columns; eluent, $0.01 \mathrm{~N} \mathrm{NaOH}$; polymer conc., ca. $1 \times 10^{-3} \mathrm{~g} \mathrm{~cm}^{-1}$; injection volume, $0.5 \mathrm{~cm}^{3}$; flow rate, $1 \mathrm{~cm}^{3} \mathrm{~min}^{-1}$. Weight-average and number-average molecular weights obtained by GPC are denoted by $M_{w}{ }^{\mathrm{GPC}}$ and $M_{n}{ }^{\mathrm{GPC}}$,
Table I. Molecular characterization of the schizopyllan samples used ${ }^{\mathrm{a}}$

\begin{tabular}{lcccc} 
Code & $M_{w} / 10^{4}$ & $M_{z} / M_{w}$ & $M_{w}{ }^{\mathrm{GPC}} / M_{n}^{\mathrm{GPC}}$ & $\frac{[\eta]}{10^{2} \mathrm{~cm}^{3} \mathrm{~g}^{-1}}$ \\
\hline $\mathrm{U}-110$ & 6.58 & 1.3 & 1.2 & $0.41_{4}$ \\
UR-28 & 13.2 & 1.2 & 1.2 & $0.64_{3}$ \\
$\mathrm{~T}-3$ & 80.0 & 1.2 & 1.2 & 9.11 \\
\hline
\end{tabular}

a Part of the data for UR-28 taken from ref 3.

respectively. The average molecular weights thus obtained along with the intrinsic viscosities $[\eta]$ in water at $25^{\circ} \mathrm{C}$ are summarized in Table I.

\section{Phase Separation Experiment}

Weighed amounts of a given sample (or samples) and water (ca. $0.5 \mathrm{~cm}^{3}$ in total) were mixed in a calibrated stoppered tube $(7 \mathrm{~mm}$ diameter, $60 \mathrm{~mm}$ long). The mixture was stirred by a magnetic stirrer bar for two days and became homogeneously turbid. It was then kept standing for more than two days in an air bath thermostatted at $25^{\circ} \mathrm{C}$. Relatively less viscous mixtures separated into two layers on standing within one day, but none of the separated layers was perfectly clear and the meniscus between the two layers remained turbid. For highly viscous mixtures, no such spontaneous separation took place. Observation by a polarizing microscope showed that the mixture formed a mosaic pattern consisting of dark and bright regions, which were found to be the isotropic and cholesteric phases, respectively. This suggests that equilibrium phase separation on a small scale is achieved in a relatively short time. However, macroscopic phase separation must be very difficult, since a large scale re-organization of highly viscous mixtures is required.

To overcome this difficulty, all the mixtures were centrifuged at $25^{\circ} \mathrm{C}$ at $c a .2100$ gravities. The centrifugation was continued until each layer became almost clear, usually within 2$3 \mathrm{~h}$. Very viscous mixtures containing a large 
fraction of sample $\mathrm{T}-3$ were centrifuged for less than $12 \mathrm{~h}$ a day and allowed to stand at $25^{\circ} \mathrm{C}$; the concentration gradient induced in each layer by the centrifugal field probably relaxed during the standing period. This procedure was repeated until the dilute layer (or layers) became almost clear. Thus, the centrifugation is expected to help an already phase-separated mixture to separate macroscopically without disturbing the phase equilibrium. It was confirmed that the phase equilibrium was not disturbed significantly by the centrifugal field unless the field was high or applied for a long time.

The volume of each separated phase was determined from its column height within $1 \%$ error, with appropriate corrections made for the volume of the magnet bar, liquid-liquid and liquid-air interfaces. The separated phases were diluted to determine their polymer concentrations by differential refractometry; the specific refractive index was taken to be 0.145 $\mathrm{cm}^{3} \mathrm{~g}^{-1}$ for $436 \mathrm{~nm}$ and $0.142 \mathrm{~cm}^{3} \mathrm{~g}^{-1}$ for $546 \mathrm{~nm} .{ }^{5}$ The solutions used for refractometry were diluted adequately to determine the intrinsic viscosities $[\eta]$. Each phase was also analyzed by GPC.

\section{RESULTS}

\section{Binary Systems}

Isotropic-cholesteric phase boundaries were determined for aqueous solutions of the three samples investigated. The results for sample UR-28 have been presented in the previous paper, ${ }^{3}$ and those for samples U-110 and T-3 are described below. As before, the polymer weight fraction is denoted by $w$ and the volume of the isotropic phase relative to the total solution volume by $\Phi$. The quantities with a prime and a double prime refer to the isotropic and cholesteric phases, respectively, and those without a prime, to the whole solution.

Figure 1 shows plots of $w$ and $[\eta]$ for each of the separated phases and the whole solution. It is seen that $w$ and $w^{\prime}$ decrease linearly with

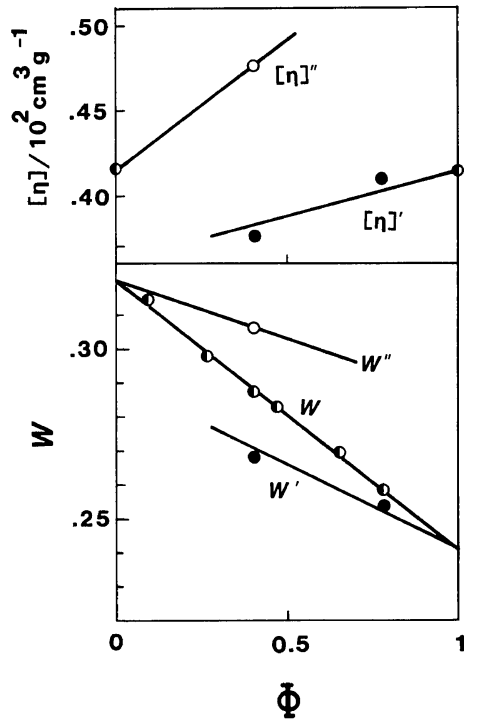

Figure 1. Phase separation data for sample U-110 at $25^{\circ} \mathrm{C}$. Unfilled circles, cholesteric phase $\left(w^{\prime \prime},[\eta]^{\prime \prime}\right)$; filled circles, isotropic phase $\left(w^{\prime},[\eta]^{\prime}\right)$; half-filled circles, the whole mixture $(w)$.

Table II. Phase separation data for the binary systems at $25^{\circ} \mathrm{C}^{\mathrm{a}}$

\begin{tabular}{|c|c|c|c|c|c|}
\hline \multirow{2}{*}{ Sample } & \multirow{2}{*}{$x$} & \multirow{2}{*}{$w^{\prime}$} & \multirow{2}{*}{$w^{\prime \prime}$} & \multirow{2}{*}{$\frac{\left(M_{w}^{\mathrm{GPC}}\right)^{\prime}}{\left(M_{n}^{\mathrm{GPC}}\right)^{\prime}}$} & \multirow{2}{*}{$\frac{\left(M_{w}{ }^{\mathrm{GPC}}\right)^{\prime \prime}}{\left(M_{w}{ }^{\mathrm{GPC}}\right)^{\prime}}$} \\
\hline & & & & & \\
\hline U-110 & 18.3 & $0.24_{1}$ & $0.32_{0}$ & 1.3 & 1.2 \\
\hline UR-28 & 36.8 & $0.18_{2}$ & $0.22_{0}$ & 1.2 & $1.1_{8}$ \\
\hline $\mathrm{T}-3$ & 223 & $0.08_{7}$ & $0.12_{0}$ & 1.2 & $1.0_{4}$ \\
\hline
\end{tabular}

a Part of the data for UR-28 taken from ref 3. $x=M_{w}$ $\left(1.67 M_{\mathrm{L}}\right) ; M_{\mathrm{L}}=2150 \mathrm{~nm}^{-1} . M_{w}^{\mathrm{GPC}} / M_{n}{ }^{\mathrm{GPC}}$, average values are given.

increasing $\Phi$ and can be extrapolated to $\Phi=0$ and 1 with common intercepts, yielding 0.320 and 0.241 for the corresponding phase boundary concentrations. Furthermore, $[\eta]^{\prime \prime}$ is larger than $[\eta]^{\prime}$ and both increase with increasing $\Phi$. This behavior of $w$ and $[\eta]$ indicates that significant molecular weight fractionation takes place upon phase separation. A similar trend of smaller extent was found for samples T-3 and UR-28. The phase separation data for these three samples are summarized in Table II. The values of $\left(M_{w}{ }^{\mathrm{GPC}}\right)^{\prime \prime} /\left(M_{w}{ }^{\mathrm{GPC}}\right)^{\prime}$ in this 
table indicate that the molecular weight fractionation is much less remarkable than predicted for polydisperse solutes with the most probable distribution. ${ }^{12}$ This finding along with the $M_{z} / M_{w}$ and $M_{w}{ }^{\mathrm{GPC}} / M_{n}{ }^{\mathrm{GPC}}$ values not much removed from unity (Table I) led us to assume that these three samples are "monodisperse" and that their aqueous solutions can be regarded as "binary."

\section{Ternary Systems}

Two systems UR-28+T-3 + water and $\mathrm{U}-110+\mathrm{T}-3+$ water were investigated. For the reasons given in the previous section, all three samples were treated as "monodisperse" polymers. The subscripts 1 and 2 refer to the lower molecular weight component (U-110 or UR-28) and higher molecular weight component $(\mathrm{T}-3)$, respectively. The weight fraction $\xi$ of component 1 in a polymer mixture was estimated from $[\eta]$ of the mixture assuming $[\eta]$ to be linearly related to $\xi$. GPC data were also used for estimating $\xi$, denoted by $\xi^{\mathrm{GPC}}$, according to the following procedure. Thus, the composition of a given solution is expressed by $w$ and $\xi$.

Figure 2 shows GPC chromatograms of the polymers in the two phases separated from a solution of UR-28+T-3 with $w=0.156$ and $\xi=0.753$, where the chromatogram calculated for the whole polymer is included for comparison. It is seen that each chromatogram contains two peaks corresponding to the two polymer components; the T-3 peak for the isotropic phase appearing as a shoulder was clearly distinguished on an LS chromatogram. It is noted that the peak position for a given component differs slightly depending on whether it is for the original polymer or for the phase-separated mixtures. A similar difference was found among the polymers recovered from biphasic mixtures of binary systems, but the chromatograms were mostly the same shape.

Therefore, each chromatogram was dissolved into two curves having essentially the same

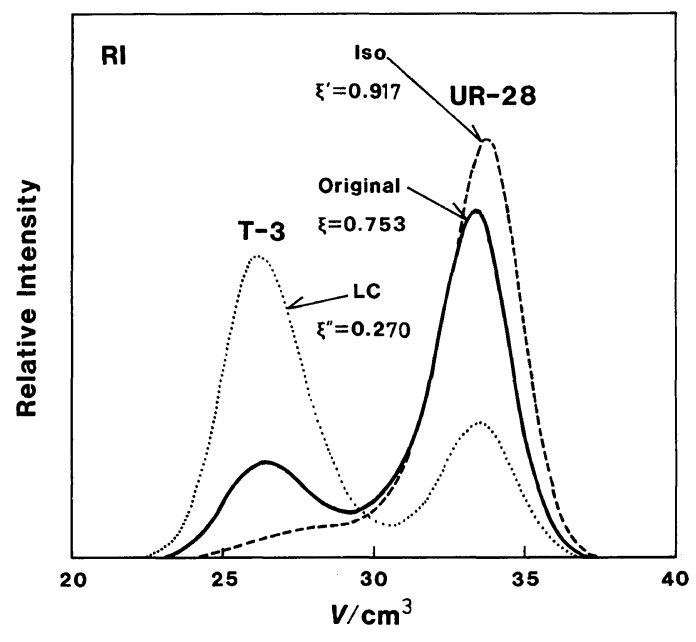

Figure 2. GPC analysis of the phase separation at $25^{\circ} \mathrm{C}$ of an original solution with $w=0.156$ and $\xi=$ 0.753 . Solid line, calculated for the polymer mixture in the original solution; dashed line, isotropic phase; dotted line, cholesteric phase.

shapes as those of the respective component polymers, and the area ratio of the two curves was used to calculate $\xi^{\mathrm{GPC}}$. The $\xi^{\mathrm{GPC}}$ values thus estimated agreed precisely with those estimated from the heights of the two peaks. This is explained by the fact that the chromatograms of either pair of U-110+T-3 and UR$28+\mathrm{T}-3$ do not overlap at their peak positions, although they are not separated completely. Thus, either or both the area and peak height was used for estimating $\xi$.

The above change in the chromatogram(s) on phase separation for each of the polymer components, as reflected in the change in $[\eta]$, is not appreciable. For this and the reasons given in the previous section, each polymer component is treated as a monodisperse, independent component in the discussion to follow.

Figure 3(a) shows a photograph of a phaseseparated solution after centrifugation, where two clear phases are separated by a narrow meniscus, allowing the volume of each phase to be determined accurately. For each solution except for the one with $\xi=0.498$, the analytical 


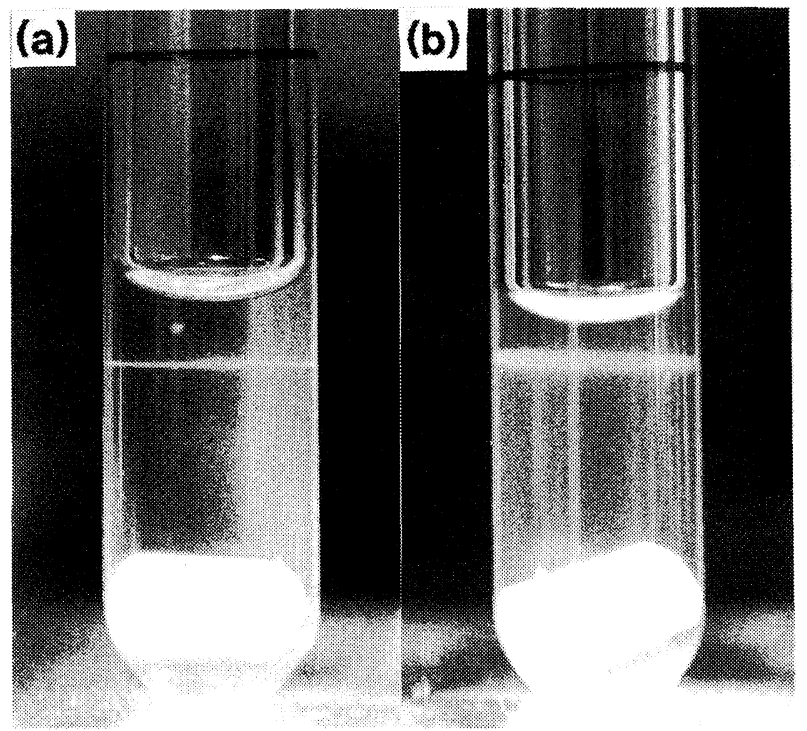

Figure 3. Biphasic separation in the ternary system UR-28+T-3+water at $25^{\circ} \mathrm{C}$. (a) $w=0.210, \xi=$ 0.950 ; (b) $w=0.228, \xi=0.751$.

results were accurate within an error of about $3 \%$ with respect to mass conservation and average intrinsic viscosity.

A solution with $w>0.2$ and $0.75<\xi<0.9$ separated on centrifugation into two transparent layers with a broad turbid meniscus between them, as shown in Figure 3(b). The volume of this meniscus portion, amounting to about $7 \%$, remained unchanged with prolonged centrifugation. This portion was separately analyzed and found to have $w$ and $\xi$ intermediate between those of the isotropic and cholesteric phases, which varied with the composition of the whole solution. Thus, we conclude that this is not a third phase but a mixture of the two phases. It is not clear why such a portion persisted.

It can be seen from Figure 2 that the solution with $\xi=0.753$ was centrifugally separated into an isotropic phase rich in component $1\left(\xi^{\prime}=0.917\right)$ and a cholesteric phase rich in component $2\left(\xi^{\prime \prime}=0.270\right)$. This fractionation according to molecular weight was observed more or less for all solutions tested.

Table III summarizes the phase separation data thus obtained for the system UR-28+T$3+$ water at $25^{\circ} \mathrm{C}$. The volume of the cholesteric phase separated from a solution with $\xi=$ 0.498 was too small to be analyzed with reasonable accuracy. Therefore, its composition was determined from that of the conjugate isotropic phase according to the lever rule. Table III shows $\xi^{\prime}$ and $\xi^{\prime \prime}$ to be in good agreement with $\left(\xi^{\mathrm{GPC}}\right)^{\prime}$ and $\left(\xi^{\mathrm{GPC}}\right)^{\prime \prime}$, respectively, and hence, whenever possible, their averages are regarded as correct. In some cases, $[\eta]^{\prime}$ was smaller than that of component 1 , owing to the polydispersity of the samples used; then $\xi^{\prime}$ was set equal to unity. Even in such cases, a small shoulder (or a small peak on an LS chromatogram) was observed, yielding $\left(\xi^{\mathrm{GPC}}\right)^{\prime}$ slightly smaller than unity.

Figure 4 shows the triangular phase diagram for the system UR-28+T-3+water at $25^{\circ} \mathrm{C}$, where each cross indicates a whole solution and a pair of unfilled circles connected by a thin line (a tie line) indicate the conjugate phases separated from it. According to the law of mass conservation, the cross must be on the tie line, and this holds with reasonable ac- 
Table III. Phase separation data for the ternary system UR-28+T-3+water at $25^{\circ} \mathrm{C}$

\begin{tabular}{|c|c|c|c|c|c|c|c|c|c|c|}
\hline \multicolumn{2}{|c|}{ Original solution } & \multirow[b]{2}{*}{$\Phi$} & \multicolumn{4}{|c|}{ Isotropic phase } & \multicolumn{4}{|c|}{ Liquid crystal phase } \\
\hline$w$ & $\xi$ & & $w^{\prime}$ & {$[\eta]^{\prime} / 10^{2} \mathrm{~cm}^{3} \mathrm{~g}^{-1}$} & $\xi^{\prime}$ & $\left(\xi^{\mathrm{GPC}}\right)^{\prime}$ & $w^{\prime \prime}$ & {$[\eta]^{\prime \prime} / 10^{2} \mathrm{~cm}^{3} \mathrm{~g}^{-1}$} & $\xi^{\prime \prime}$ & $\left(\xi^{\mathrm{GPC}}\right)^{\prime \prime}$ \\
\hline 0.121 & 0.498 & 0.85 & 0.112 & $4.0_{2}$ & $0.60_{1}$ & $0.56_{3}$ & 0.148 & $\left(6.7_{6}\right)^{\mathrm{a}}$ & $\left(0.27_{8}\right)^{\mathrm{a}}$ & - \\
\hline 0.156 & 0.753 & $0.80_{6}$ & 0.145 & $1.0_{9}$ & 0.95 & $0.91_{7}$ & 0.197 & 7.6 & 0.18 & $0.27_{0}$ \\
\hline 0.204 & 0.751 & 0.52 & 0.180 & $0.64_{2}$ & 1 & $0.99_{2}$ & 0.236 & $4.3_{5}$ & $0.56_{2}$ & $0.56_{5}$ \\
\hline 0.215 & 0.803 & $0.33_{9}$ & 0.189 & $0.61_{6}$ & 1 & $0.99_{0}$ & 0.239 & $3.0_{5}$ & $0.71_{6}$ & $0.72_{2}$ \\
\hline 0.210 & 0.896 & $0.39_{0}$ & 0.183 & $0.60_{9}$ & 1 & $0.99_{6}$ & 0.229 & $2.0_{1}$ & $0.83_{9}$ & - \\
\hline 0.210 & 0.950 & $0.25_{3}$ & 0.188 & $0.56_{3}$ & 1 & $0.99_{8}$ & 0.227 & $1.1_{6}$ & $0.93_{9}$ & $0.93_{4}$ \\
\hline
\end{tabular}

a Estimated from the data for the isotropic phase according to the lever rule.

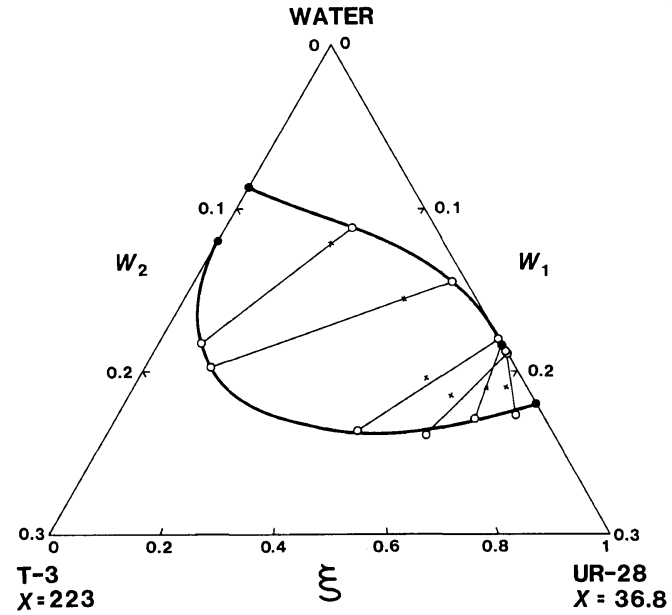

Figure 4. Binodal for the system UR-28+T-3+water at $25^{\circ} \mathrm{C}$. Crosses, original mixtures; unfilled circles, separated solutions; filled circles; phase boundaries for the respective binary systems; $\xi=w_{1} /\left(w_{1}+w_{2}\right)$.

curacy for most cases tested. The thick solid curves drawn to fit the unfilled circles are the binodal for this system. The filled circles on the polymer-solvent axes indicate the phase boundary concentrations for the corresponding binary systems: UR-28 + water and T-3+ water. Two of the unfilled circles are seen between the filled circles, reflecting the polydispersity of sample UR-28.

Figure 4 shows that pronounced molecular weight fractionation occurs on phase separation. It is particularly remarkable for solutions with $\xi>0.75$, where the higher molecular weight component is almost excluded from the isotropic phase.

We now describe the results for the system U-110+T-3 + water. After 2-3h's centrifugation, a mixture separated into a transparent upper layer and a lower layer remaining turbidly white. On further centrifugation, the lower layer became less turbid from the bottom but the interface between the two layers become more turbid. Since it was difficult to make the lower layer perfectly clear, each phase was analyzed after about $15 \mathrm{~h}$. The results are summarized in Table IV.

Three solutions in the composition range $\xi>0.8$ and $0.29<w<0.32$ separated into three phases. Figure 5 shows a photograph of such a phase-separated solution. As seen here, the lowest layer was turbid in every case studied, as was the cholesteric phase in the two phase separation. Observed between crossed polars, the uppermost phase was dark and identified to be an isotropic phase $\left(\mathrm{I}_{0}\right)$, while the middle phase showed iridescent colors and was a cholesteric phase. The middle phase was designated cholesteric $2\left(\mathrm{~A}_{2}\right)$ and the lowermost phase, cholesteric $1\left(\mathrm{~A}_{1}\right)$. Two other solutions separated in a similar manner into three phases. The analytical results are summarized in Table $\mathrm{V}$.

In no case with the three phase separation was it easy to determine the composition of the smallest volume phase, but those of the rest were determined with reasonable accuracy. A comparison of the results obtained accurately 
Multi-Phase Equilibrium in Aqueous Schizophyllan

Table IV. Phase separation data for the ternary system U-110+T-3 + water at $25^{\circ} \mathrm{C}$

\begin{tabular}{|c|c|c|c|c|c|c|c|c|c|c|}
\hline \multicolumn{2}{|c|}{ Original solution } & \multirow[b]{2}{*}{$\Phi$} & \multicolumn{4}{|c|}{ Isotropic phase } & \multicolumn{4}{|c|}{ Liquid crystal phase } \\
\hline$w$ & $\xi$ & & $w^{\prime}$ & {$[\eta]^{\prime} / 10^{2} \mathrm{~cm}^{3} \mathrm{~g}^{-1}$} & $\xi^{\prime}$ & $\left(\xi^{\mathrm{GPC}}\right)^{\prime}$ & $w^{\prime \prime}$ & {$[\eta]^{\prime \prime} / 10^{2} \mathrm{~cm}^{3} \mathrm{~g}^{-1}$} & $\xi^{\prime \prime}$ & $\left(\xi^{\mathrm{GPC}}\right)^{\prime \prime}$ \\
\hline 0.137 & 0.500 & $0.85_{8}$ & 0.128 & $3.5_{7}$ & $0.63_{7}$ & $0.55_{4}$ & 0.169 & 8.4 & 0.082 & $0.24_{3}$ \\
\hline 0.200 & 0.747 & $0.80_{8}$ & 0.183 & $0.49_{6}$ & $0.99_{1}$ & 1 & 0.292 & $7.7_{4}$ & $0.15_{8}$ & $0.15_{5}$ \\
\hline 0.281 & 0.750 & $0.67_{8}$ & 0.252 & $0.40_{5}$ & 1 & 1 & 0.349 & $5.8^{4}$ & $0.38^{\circ}$ & $0.39_{7}$ \\
\hline
\end{tabular}

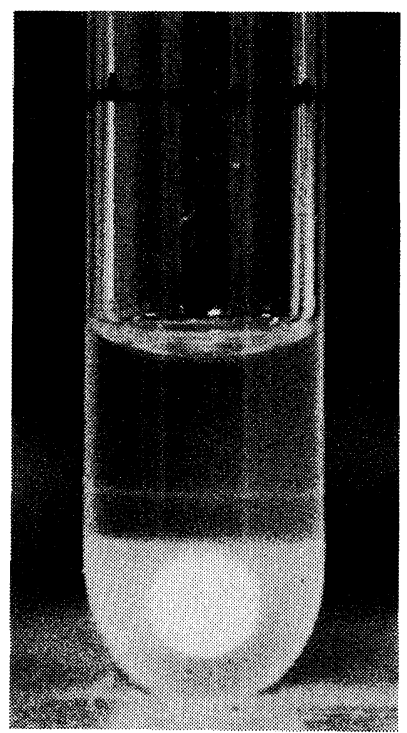

Figure 5. Triphase separation in the ternary system U$110+\mathrm{T}-3+$ water at $25^{\circ} \mathrm{C}$. Solution (i): $w=0.290, \xi=$ 0.840 .

shows that the composition of a given type of phase $\left(I_{0}, A_{1}\right.$, or $\left.A_{2}\right)$ is nearly the same irrespective of the original solution. For example, the composition of the isotropic phase is essentially the same for the three solutions tested. The compositions of the three coexisting phases averaged over the results accurately obtained are given in Table VI.

The solution with $w=0.373$ and $\xi=0.655$ separated into two layers: a clear upper layer and a turbidly white lower layer. Observation between crossed polars indicated both layers to be essentially cholesteric. The compositions of the separated phases are also included in Table V.

The results from the phase separation ex-
Table V. Analysis of the three coexisting phases in the system U-110+T-3+ water at $25^{\circ} \mathrm{C}^{*}$

\begin{tabular}{|c|c|c|c|}
\hline & $\begin{array}{c}\text { Isotropic } \\
\text { phase } \\
\mathrm{I}_{0}\end{array}$ & $\begin{array}{c}\text { Cholesteric } \\
\text { phase } \\
\mathrm{A}_{2}\end{array}$ & $\begin{array}{c}\text { Cholesteric } \\
\text { phase } \\
\mathrm{A}_{1}\end{array}$ \\
\hline \multicolumn{4}{|c|}{ (i) $w=0.290, \xi=0.840$} \\
\hline volume ratio & $0.54_{5}$ & $\left(0.19_{3}\right)^{a}$ & $0.26_{2}$ \\
\hline$w$ & 0.264 & 0.303 & 0.356 \\
\hline$[\eta] / 10^{2} \mathrm{~cm}^{3} \mathrm{~g}^{-1}$ & 0.39 & $0.77_{2}$ & 4.6 \\
\hline$\xi$ & 1 & $0.95_{9}$ & $0.51_{9}$ \\
\hline$\xi^{\mathrm{GPC}}$ & $0.97_{6}$ & $\left(0.96_{1}\right)$ & $0.50_{5}$ \\
\hline \multicolumn{4}{|c|}{ (ii) $w=0.317, \xi=0.841$} \\
\hline volume ratio & $0.22_{4}$ & $0.53_{7}$ & $0.23_{9}$ \\
\hline$w$ & 0.281 & 0.319 & 0.360 \\
\hline$[\eta] / 10^{2} \mathrm{~cm}^{3} \mathrm{~g}^{-1}$ & 0.36 & $0.58_{4}$ & $4.9_{5}$ \\
\hline$\xi$ & 1 & $0.98_{0}$ & $0.47_{8}$ \\
\hline$\xi^{\mathrm{GPC}}$ & - & 1 & - \\
\hline \multicolumn{4}{|c|}{ (iii) $w=0.299, \xi=0.901$} \\
\hline volume ratio & $0.36_{9}$ & $0.4_{5}$ & $\left(0.1_{8}\right)^{a}$ \\
\hline$w$ & 0.270 & 0.318 & 0.378 \\
\hline$[\eta] / 10^{2} \mathrm{~cm}^{3} \mathrm{~g}^{-1}$ & $0.38_{1}$ & $0.58_{6}$ & 3.3 \\
\hline$\xi$ & 1 & $0.98_{0}$ & 0.67 \\
\hline$\xi^{\mathrm{GPC}}$ & 1 & $0.98_{2}$ & $\left(0.66_{3} \backslash\right.$ \\
\hline \multicolumn{4}{|c|}{ (iv) $w=0.373, \xi=0.655$} \\
\hline volume ratio & 0 & $0.50_{4}$ & $0.49_{6}$ \\
\hline$w$ & - & 0.351 & 0.417 \\
\hline$[\eta] / 10^{2} \mathrm{~cm}^{3} \mathrm{~g}^{-1}$ & - & $0.53_{2}$ & $5.9_{7}$ \\
\hline$\xi$ & - & $0.98_{6}$ & $0.36_{1}$ \\
\hline
\end{tabular}

* Solution (iv) separated into two cholesteric phases.

a Values in parentheses are less accurate for reason given in the text.

periments for the system U-110+T-3 + water are illustrated in a ternary phase diagram in Figure 6. The half-filled circles represent the compositions of the phases separated on the three phase equilibrium. For example, solution (ii) indicated by the cross $\mathrm{A}_{0}$ inside the triangle separated into three phases, A, B, and C. The 
Table VI. Composition of the three coexisting phases in the system U-110+T-3+ water at $25^{\circ} \mathrm{C}$

\begin{tabular}{cccc}
\hline & $\mathrm{I}_{0}$ & $\mathrm{~A}_{2}$ & $\mathrm{~A}_{1}$ \\
\hline$w$ & 0.272 & 0.319 & 0.358 \\
$\xi$ & $0.99_{5}$ & $0.98_{6}$ & $0.50_{1}$ \\
\hline
\end{tabular}

data points for the isotropic phase $\left(\mathrm{I}_{0}\right)$ and cholesteric $2\left(\mathrm{~A}_{2}\right)$ are located very close to the water-U-110 axis, indicating that component 2 is nearly excluded from these phases. The viscosity of cholesteric 1 was much higher than those of the others, because component 2 was contained exclusively in it.

It is seen that the cholesteric-cholesteric phase separation occurred in a relatively wide region below the triphasic region. It is suggested that the dilute and concentrated phases correspond to $A_{2}$ and $A_{1}$ phases in the triphase equilibrium, respectively.

\section{DISCUSSION}

\section{Theory of Abe and Flory for ternary Athermal} Solutions

Abe and Flory ${ }^{11}$ extended the original Flory theory for rodlike polymers ${ }^{8}$ to a ternary system containing two monodisperse rodlike polymers of different lengths. We attempted to see if their theoretical prediction was comparable to our present data. Actually, this theory is concerned with nematic liquid crystals, whereas aqueous schizophyllan forms a cholesteric mesophase. Furthermore, the theoretical ternary system consists of monodisperse polymers, whereas the experimental ones contain polydisperse polymers, although well fractionated. For the moment, we neglect these differences.

The Abe-Flory theory is formulated in terms of four variables, the volume fraction $\phi_{i}$ and axial ratio $x_{i}$ of polymer species $i(i=1,2)$. Alternatively, we take $x_{1}, x_{2}, \phi=\phi_{1}+\phi_{2}$, and $\xi=\phi_{1} / \phi$ as the necessary variables. As shown

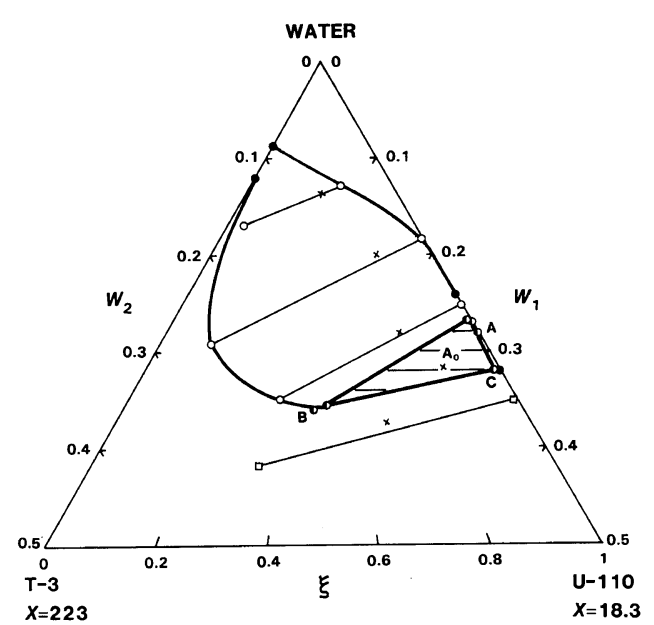

Figure 6. Ternary phase diagram for the system U$110+\mathrm{T}-3+$ water at $25^{\circ} \mathrm{C}$. Half-filled circles, solutions from the triphase equilibrium; hatched triangle, region for the triphase equilibrium; unfilled squares, solutions separated from a cholesteric-cholesteric mixture. A, B, and $C$ : three conjugate phases separated from $A_{0}$ corresponding to solution (ii) in Table $\mathrm{V}$.

in the previous section, the experimental data are presented in terms of $w, M_{w}$, and $\xi$. To make a comparison of theory with experiment, these experimental variables must be converted to theoretical ones. This was done as follows using the relations:

$$
\begin{aligned}
& \phi=v_{\mathrm{p}} w /\left[v_{\mathrm{p}} w+(1-w) v_{0}\right] \\
& x=M_{w} /\left(M_{\mathrm{L}} d\right)
\end{aligned}
$$

where $v_{\mathrm{p}}$ is the partial specific volume of schizophyllan in water and $v_{0}$, the specific volume of water at $25^{\circ} \mathrm{C}, M_{\mathrm{L}}$, the molecular weight per unit length of the helix, and $d$, the helix diameter. $M_{\mathrm{L}}$ and $v_{\mathrm{p}}$ were taken to be $2150 \mathrm{~nm}^{-1}$ and $0.619 \mathrm{~cm}^{3} \mathrm{~g}^{-1}$, respectively. ${ }^{5,6}$ The helix diameter consistent with these $M_{\mathrm{L}}$ and $v_{\mathrm{p}}$ was calculated to be $1.67 \mathrm{~nm} .^{3}$ Thus, $x$ was calculated from $x=M_{w} /\left(1.67 M_{\mathrm{L}}\right)$.

Figure 7 shows a theoretical phase diagram calculated for the axial ratios corresponding to the polymer combination UR-28+T-3. Here, the thick lines are phase boundaries and the thin straight lines are tie lines connecting the conjugate phases, and arrows indicate the 


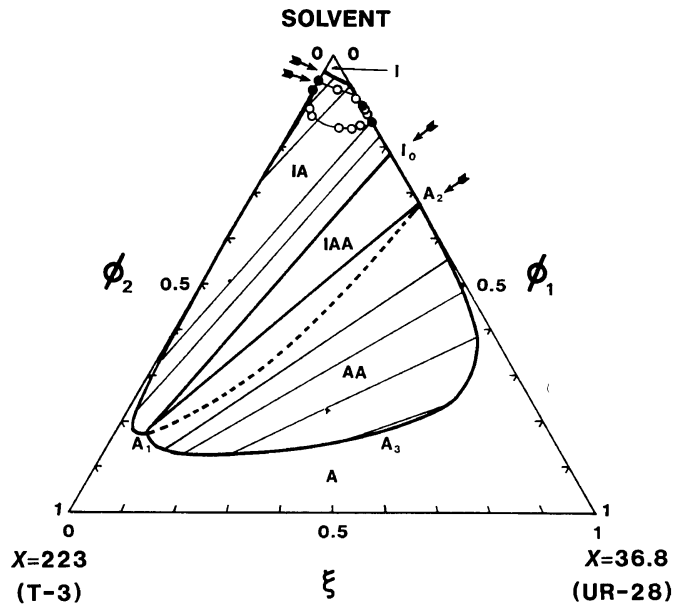

Figure 7. Theoretical phase diagram calculated according to Abe and Flory ${ }^{11}$ for an athermal system with $x=36.8$ and 223. Circles, data points; arrows, theoretical phase boundaries for the binary systems. I, isotropic; IA, isotropic-anisotropic; IAA, isotropic-anisotropicanisotropic; AA, anisotropic-anisotropic; A, anisotropic regions. Dashed line, anisotropic-anisotropic phase boundary due to the Abe-Flory calculation (see the text).

phase boundary concentrations for the respective binary systems. This phase diagram consists of five regions including the isotropic (I) and anisotropic (A) regions. From this phase diagram along with those calculated by Abe and Flory, we may summarize the features of the phase behavior of this kind of ternary system as follows.

(1) There is a wide biphasic region extending to a high concentration along the solventcomponent 2 axis (region IA).

(2) Below this biphasic region, there exists a wide triphasic region indicated by a triangle $\mathrm{I}_{0} \mathrm{~A}_{1} \mathrm{~A}_{2}$ (region IAA), where two anisotropic phases and an isotropic phase coexist at equilibrium.

(3) In either of the two regions, pronounced fractionation according to rod length occurs on phase separation. Indeed, the larger component is almost completely excluded from the dilute phase (or phases). The concentration difference between the conjugate dilute and concentrated phases is very large.

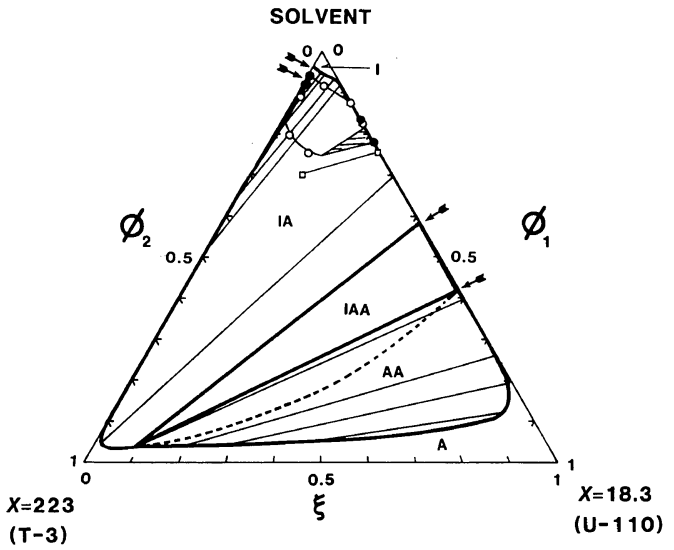

Figure 8. Theoretical phase diagram for an athermal system with $x=18.3$ and 223 .

(4) Below the triphasic region appears another biphasic region $\mathrm{AA}$ encompassed by a curve $A_{1} A_{2} A_{3}$, where two anisotropic phases coexist. The dashed line due to the Abe-Flory calculation is shown to be the locus of the termini of the tie lines ending near point $\mathrm{I}_{0}$. The equilibrium phase boundary is the curve $\mathrm{A}_{1} \mathrm{~A}_{3} \mathrm{~A}_{2}$ with the tie lines indicated. The concentration difference between the two conjugate phases is smaller and molecular weight fractionation is less conspicuous than in the other regions.

(5) The immiscibility gap containing the three regions widens as the ratio $x_{2} / x_{1}$ increases as shown in Figure 8.

(6) For smaller $x_{2} / x_{1}$, there is no triphasic region nor biphasic region for two anisotropic phases.

Recently, Lekkerkerker et al. ${ }^{15}$ extended the Onsager theory for monodisperse rods to the ternary system considered here and calculated ternary phase diagrams. However, they found no triphase separation for $x_{2} / x_{1}=5$, for which the Abe-Flory theory predicts its existence. It is not clear whether this is essential to the extended Onsager theory or the rod length ratio is still too small.

The theoretical predictions summarized above are compared with the experimental phase diagrams shown in Figures 4 and 6 in 
the following.

As predicted in feature (1), either of the experimental phase diagrams contains a wide biphasic region for the isotropic and cholesteric phases, region IA. It is seen that in most cases, component 2 is almost completely excluded from the dilute isotropic phase, which conforms to feature (3).

The phase diagram for the system U-110+ $\mathrm{T}-3+$ water has a triphasic region, where two cholesteric phases and one isotropic phase coexist at equilibrium (region IAA); the two dilute phases, isotropic and cholesteric, are almost devoid of component 2 . This is precisely what is predicted in features (2) and (3).

Below the triphasic region, there are a pair of data points for the cholesteric-cholesteric phase separation with a relatively small concentration difference between the conjugate phases. This result may be compatible with feature (4), although the precise shape of this biphasic region (region AA) is yet to be elucidated.

Figures 4 and 6 show that the immiscibility gap is smaller for the system UR-28+T-3+ water than for the system $\mathrm{U}-110+\mathrm{T}-3$ + water, and the former has no region corresponding to regions IAA and $\mathrm{AA}$, which conforms to features (5) and (6), because $x_{2} / x_{1}$ is small for the former system.

In sum, the present data for aqueous schizophyllan are quite consistent with the Abe-Flory theory for ternary solutions of rodlike polymers, exemplifying many important qualitative features of the theory.

\section{Data for Other Rodlike Polymers}

Recently, Sasaki et al. ${ }^{21}$ reported phase equilibrium data for solutions of poly $(\gamma$-benzyl L-glutamate) (PBLG) in benzyl alcohol using two PBLG samples of different molecular weights. Their data give a phase diagram similar to that shown in Figure 4. They found no triphase separation. These results are consistent with the Abe-Flory prediction (1), (3), and (5) and our experimental results, because the molecular weight ratio of the two samples is only about 3 .

Polyisocyanates form liquid crystal phases under appropriate conditions. ${ }^{24}$ Aharoni and Walsh ${ }^{19,20}$ and Conio et al. ${ }^{22}$ studied the phase separation of the solutions of these polymers. A single sample or combinations of more than two samples with different molecular weights, all polydisperse, were used and significant molecular weight fractionation was found to occur on phase separation. They have taken these findings to substantiate the validity of the theories for polydisperse rods. ${ }^{12-14}$

However, the experimental data for PBLG and polyisocyanates quoted here may not be compared directly with the Abe-Flory prediction, because all the samples used appear to be considerably polydisperse and not to cover a molecular weight range appropriate for it, and also because there is ample evidence $22,25,26$ that polyisocyanates should be regarded as semi-flexible.

\section{Quantitative Comparison}

In spite of the excellent qualitative agreement between theory and experiment noted above for aqueous schizophyllan, the large discrepancy between them may be noted when the experimental data indicated by circles are compared with the theoretical prediction in Figures 7 and 8. Indeed, in Figure 7, the experimental binodal is confined in the range $\phi<0.2$, while the theory predicts an immiscibility gap extending down to $\phi=0.8$. In addition, the molecular weight fractionation is extremely conspicuous in the theory; in the isotropic-anisotropic boundary near point $\mathrm{I}_{0}$, $1-\xi$ is predicted to be as small as of the order of $10^{-5}$ in comparison with the experimental values of about $10^{-3}$.

Figure 8 shows that the quantitative discrepancy is even more remarkable for the system $\mathrm{U}-110+\mathrm{T}-3$ + water than for the former system. Here, the theory predicts a wide triphasic region extending to $1-\xi=0.9$, while the experimental triangle is confined below $1-\xi$ of 


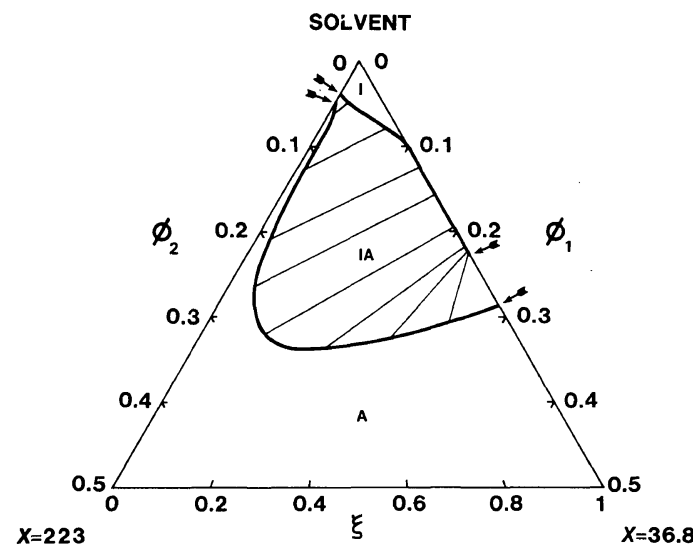

Figure 9. Theoretical phase diagram calculated for $x=$ 36.8 and 223 with $\chi=-0.26$.

0.5 and thus much narrower than the theoretical triangle.

We may point out two important reasons for the discrepancy found here.

(1) As discussed in the previous paper, ${ }^{3}$ binary phase diagrams of aqueous schizophyllan cannot be described correctly by the original Flory theory, and hence by the Abe-Flory theory. This can be seen from the experimental phase boundaries (filled circles) which do not agree with the theoretical ones indicated by arrows in Figures 7 and 8.

(2) The Abe-Flory theory is concerned with athermal solutions in the sense that the interaction parameter $\chi$ is not involved. This is in contrast to the recent finding ${ }^{3}$ that aqueous schizophyllan is non-athermal and can be characterized by a negative $\chi$ approximately linear in $\phi$.

\section{Ternary Phase Diagrams Corrected for the} Non-Athermal Effect

We attempted to correct the Abe-Flory theory for the non-athermal nature of the system. We calculated phase diagrams according to Abe and Flory using the relevant chemical potential expressions modified to have an additional term involving $\chi ; \chi$ was taken to be -0.26 , the value for the constant term in the experimental expression. ${ }^{3}$

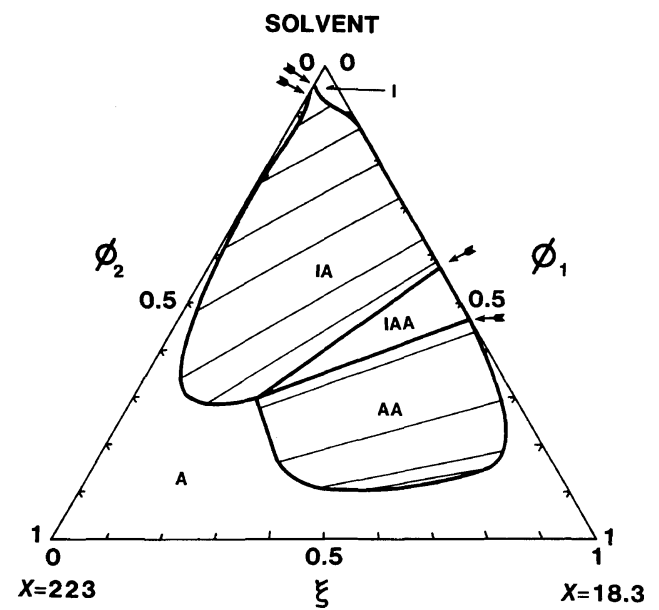

Figure 10. Theoretical phase diagram calculated for $x=18.3$ and 223 with $\chi=-0.26$.

Figure 9 shows the phase diagram thus calculated for the system UR-28+T-3 + water. The most striking change induced by $\chi$ is the disappearance of regions IAA and AA, which is in agreement with the experimental data. The entire immiscibility gap is narrower in this non-athermal case than in the athermal case (Figure 7). Sasaki et al. ${ }^{21}$ attempted a similar modification of the Abe-Flory theory and showed the biphasic gap to be narrowed by such a modification. However, their phase diagram contained no triphase region because the length ratio of the two polymer components was small.

Figure 10 shows similar results for the system $\mathrm{U}-110+\mathrm{T}-3+$ water. Both the biphasic and triphasic regions are narrowed by introducing a negative $\chi$. The region for the anisotropic-anisotropic phases (region AA) has a peculiar shape extending deeply into the anisotropic region. The entire phase diagram resembles closer the experimental one than does the athermal one shown in Figure 8.

In either case discussed above, however, quantitative agreement between theory and experiment is still far from satisfactory. This is mainly due to the first reason mentioned above. An attempt at solving this disagreement by adjusting the axial ratios of the polymer 
components so as to fit the data for the binary systems failed, because the calculated phase diagram had no triphasic region. The need for further theoretical study is indicated.

Acknowledgments. The authors thank Professor T. Fujimoto and Dr. Y. Miyaki for assisting them with the GPC measurements.

\section{REFERENCES}

1. K. Van, T. Norisuye, and A. Teramoto, Mol. Cryst. Liq. Cryst., 78, 123 (1981).

2. K. Van and A. Teramoto, Polym. J., 14, 999 (1982).

3. T. Itou, K. Van, and A. Teramoto, J. Appl. Polym. Sci., in press.

4. K. Van, T. Asakawa, and A. Teramoto, Polym. J., 16, 61 (1984).

5. T. Norisuye, T. Yanaki, and H. Fujita, J. Polym. Sci., Polym. Phys. Ed., 18, 547 (1980).

6. T. Yanaki, T. Norisuye, and H. Fujita, Macromolecules, 13, 1462 (1980).

7. L. Onsager, Ann. N. Y. Acad. Sci., 51, 627 (1949).

8. P. J. Flory, Proc. R. Soc. London, Ser. A, 234, 73 (1956).

9. P. J. Flory and G. Ronca, Mol. Cryst. Liq. Cryst., 54, 289 (1979).

10. T. Itou and A. Teramoto, Macromolecules, 17, 1419 (1984).
11. A. Abe and P. J. Flory, Macromolecules, 11, 1122 (1978).

12. P. J. Flory and R. S. Frost, Macromolecules, 11, 1126 (1978).

13. R. S. Frost and P. J. Flory, Macromolecules, 11, 1134 (1978).

14. J. K. Moscicki and G. Williams, Polymer, 23, 558 (1982).

15. H. N. W. Lekkerkerker, Ph. Coulon, and V. D. Haegen, J. Chem. Phys., 80, 3427 (1984).

16. S. L. Kwolek, P. W. Morgan, J. R. Schaefgen, and L. W. Gulrich, Macromolecules, 10, 1390 (1977).

17. G. Conio, E. Bianchi, A. Ciferri, and A. Tealdi, Macromolecules, 14, 1084 (1981).

18. W. G. Miller, C. C. Wu, E. L. Wee, G. L. Santee, J. H. Rai, and K. G. Goebel, Pure Appl. Chem., 38, 37 (1974).

19. S. M. Aharoni and E. K. Walsh, Macromolecules, 12, 271 (1979).

20. S. M. Aharoni, Polym. Bull., 9, 186 (1983).

21. S. Sasaki, K. Tokuma, and I. Uematsu, Polym. Bull., 10, 539 (1983).

22. G. Conio, E. Bianchi, A. Ciferri, and W. R. Krigbaum, Macromolecules, 17, 856 (1984).

23. This operation was made to separate the main fraction from low molecular weight contaminants by phase separation.

24. S. M. Aharoni, Macromolecules, 12, 94 (1979).

25. L. J. Fetters and H. Yu, Macromolecules, 4, 385 (1971).

26. H. Murakami, T. Norisuye, and H. Fujita, Macromolecules, 13, 345 (1980). 